\title{
Estudio preliminar para el diseño y validación de un cuestionario sobre la percepción de la seguridad según el sistema antivuelco de las porterías en eventos deportivos
}

\author{
Preliminary Study of Design and Validation of a Questionnaire on the Perception \\ of Safety According the Goalpost Anti-Tip System in Sports Events
}

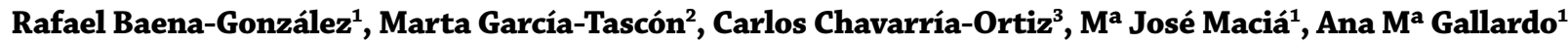 \\ 1 Facultad de Deporte. Universidad Católica de Murcia. España. \\ 2 Facultad Ciencias del Deporte. Universidad Pablo de Olavide. Sevilla. España. \\ 3 Escuela Universitaria de Osuna (Centro adscrito de la Universidad de Sevilla). Sevilla. España.
}

CORRESPONDENCIA:

Marta García Tascón

margata@upo.es

Recepción: noviembre 2020 • Aceptación: enero 2021
CÓMO CITAR EL ARTÍCULO:

Baena-González, R., García-Tascón, M., Chavarría-Ortiz, C., Maciá, M. J. \& Gallardo, A. M. (2021). Estudio preliminar para el diseño y validación de un cuestionario sobre la percepción de la seguridad según el sistema antivuelco de las porterías en eventos deportivos. Cultura, Ciencia y Deporte, 16(50), 583-592. http://dx.doi. org/10.12800/ccd.v16i50.1613

\section{Resumen}

En los eventos deportivos la seguridad de los deportistas es fundamental y el sistema antivuelco de las porterías es un elemento imprescindible para evitar lesiones y accidentes en el balonmano/futsal. El objetivo de este estudio fue realizar un estudio preliminar del diseño y validación de un nuevo cuestionario para conocer la opinión de los agentes implicados en los eventos deportivos acerca de la percepción en el rendimiento del juego del balonmano/futsal del sistema antivuelco de las porterías. La validación de contenido se ha llevado a cabo a través de dieciséis expertos mediante entrevista estructurada y la validez de comprensión determinada por la aplicación del cuestionario a una muestra de 60 participantes en el Torneo Internacional 4 Naciones de Balonmano en el año 2019. El alfa de Cronbach sobre la base de 18 ítems es de .860 en una escala 0-1, lo que indica una consistencia interna adecuada de los ítems, la prueba $x^{2}$ de Friedman es de $p<.05$, lo cual indica que de forma individual cada pregunta incluida en el instrumento aporta información relevante. Este estudio preliminar indica que el instrumento podría considerarse válido y fiable para valorar la opinión de los agentes deportivos sobre el sistema antivuelco de las porterías.

Palabras clave: Antivuelco, cuestionario, seguridad, niños, eventos deportivos.

\section{Abstract}

The safety of the athletes is basic at sports events and the anti-tip system of the goalposts is an essential element to avoid injuries and accidents in handball/ futsal. The objective of this study was to carry out a preliminary study of the design and validation of a new questionnaire to know the opinion of sports agents involved in sports events about the perception of the performance in the handball/futsal game with the goalpost anti-tip system. The content validation was carried out by means of a structured interview with 16 experts and the understanding validity to a sample of 60 participants in the 4 Nations International Handball Tournament held in 2019. Cronbach's alpha on the items is .860 on a scale $0-1$, which indicates an adequate internal consistency of the items and Friedman's $x 2$ test is $p<.05$, which indicates that individually each included question in the instrument provides relevant information, so this preliminary study indicates that the instrument could be considered valid and reliable to assess the opinion of sports agents on the goalpost anti-tip system.

Key words: Anti-tip, questionnaire, safety, children, sports events. 


\section{Introducción}

La seguridad es fundamental en muchos deportes y frecuentemente las reglas son creadas con el objetivo de evitar serios accidentes y lesiones (Wright, 2014). Prevenir accidentes y lesiones en los eventos deportivos es una prioridad para las organizaciones deportivas y el equipamiento usado es vital para ello en muchas ocasiones (Andrew et al., 2003; Krauss, 2004). En este sentido, velar por la seguridad e integridad de personas, instalaciones, espectadores, participantes y equipamientos forma parte del plan estratégico de la organización de un evento deportivo (Magaz-González \& Fanjul-Suárez, 2012).

Según la Comisión Europea, 7000 personas fallecieron a causa de accidentes o lesiones derivados del deporte entre 2010 y 2012 (Kisser \& Bauer, 2012), no especificando la cantidad de muertes en relación a las instalaciones o equipamientos deportivos, es decir, no existe un registro oficial (Baena-González et al., 2020a). En este sentido, la investigación sobre accidentalidad en los eventos deportivos en España es escasa y se obtiene realizando búsquedas en las publicaciones de los medios de comunicación (García-Tascón, 2018a; Gavilán, 2011). Por este motivo adquiere especial relevancia prestar atención a los estudios sobre la accidentabilidad y la propensión al accidente de los participantes en los eventos deportivos (Babí et al., 2018), así como al aumento de las noticias de accidentes en espacios deportivos y sobre la seguridad de los mismos, especialmente cuando intervienen niños (García-Tascón, 2014). Desde el año 2000, se registra la cifra de 18 menores fallecidos en accidentes con porterías o canastas de instalaciones deportivas, parques o centros escolares (García-Tascón, 2018b). En Alemania entre los años 1996 y 2013 fallecieron 6 niños y 8 más en países de centro Europa (Katthage $\&$ Thieme-Hack, 2013).

Las innovaciones tecnológicas aplicadas a los equipamientos deportivos utilizados en los eventos deportivos y/o los cambios en las reglas que rigen las diferentes modalidades deportivas ayudan a disminuir el riesgo de accidentes y lesiones (Krauss, 2004; Laferrier et al., 2012; Macan et al., 2006; Ratten, 2019). En este sentido, existen varios estudios que evalúan la influencia de los equipamientos deportivos en el rendimiento deportivo (Angulo et al., 2019; Baena-González et al., 2020a; Baena-González et al., 2020b; Dosseville, 2007; Giatsis \& Tzetzis, 2003; O’Donoghue, 2012; Ortega et al., 2008a; Ronglan \& Grydeland, 2006; Williams et al., 2005), e incluso algunas de las propuestas han supuesto la creación, adaptación o ampliación de las reglas específicas de un determinado deporte (Baena-González et al., 2020a; Baena-González et al., 2020b; Heino, 2000; Ortega et al., 2012a; Vizcaíno et al., 2013).

Por su parte, las organizaciones deportivas son en parte responsables de los daños que se originan en los eventos deportivos. Así lo indica el art. 63.1 de la Ley 53/2002 de Medidas Fiscales, Administrativas y del Orden Social:

"Las personas físicas o jurídicas que organicen cualquier prueba, competición o espectáculo deportivo de ámbito estatal o los eventos que constituyan o formen parte de dichas competiciones serán responsables de los daños y desórdenes que pudiera producirse por su falta de diligencia o prevención, todo ello de conformidad $y$ con el alcance que se prevé en los Convenios Internacionales sobre la violencia deportiva ratificados por España. Esta responsabilidad es independiente de la que pudieran haber incurrido en el ámbito penal o en el puramente deportivo como consecuencia de su comportamiento en la propia competición".

Las organizaciones deportivas deben adoptar medidas oportunas para proteger a los deportistas y evitar que se produzcan accidentes y lesiones (Cherington, 2001; Wright, 2014). De este modo, la EHF (European Handball Federation) publicó un estudio referente a las medidas a tomar para prevenir lesiones de los jugadores de balonmano y, entre ellas, menciona la adaptación de equipamientos e instalaciones (European Association for Injury Prevention and Safety Promotion, 2010). Concretamente, la IHF (International Handball Federation), organismo que afecta a 209 países en todo el mundo, modificó la regla 1:2 del reglamento oficial (1 de julio de 2019) referente al sistema antivuelco de las porterías. Anteriormente, indicaba que las porterías deben estar firmemente ancladas al suelo o a la pared trasera, circunstancia que no siempre es posible porque el pavimento de la instalación no está acondicionado para ello (Baena-González et al., 2020a; Baena-González et al., 2020b; IHF, 2019). El cambio federativo ha sido fundamental para posibilitar el uso de diferentes sistemas antivuelco en las porterias y en su actual versión añade: "o provistas con un sistema antivuelco. Esta nueva regla se incorpora con el objetivo de evitar accidentes" (IHF, 2019:5).

El sistema antivuelco es el mecanismo que proporciona estabilidad y evita el vuelco de la portería (BaenaGonzález et al., 2020a; Baena-González et al., 2020b). Es un equipamiento deportivo imprescindible para la práctica del balonmano, hockey o fútbol, y presente en multitud de eventos deportivos. En este sentido, se considera un elemento fundamental para evitar acci- 
dentes, cuya ausencia, estado y uso de este es evaluado en estudios referentes a los riesgos presentes en las instalaciones deportivas (del Campo \& Sánchez, 2016; Herrador-Sánchez \& García-Tascón, 2016; García-Tascón et al., 2014; Latorre et al., 2012; Montalvo et al., 2010). Además, existen herramientas para evaluar las características de las instalaciones y equipamientos deportivos, como el cuestionario de Latorre (2008), o tomando como base la normativa NIDE, normativa sobre instalaciones deportivas y de esparcimiento, así como a las EN, normas europeas (del Campo \& Sánchez, 2016; GarcíaTascón et al., 2014; Montalvo et al., 2010).

Del mismo modo, la opinión de los entrenadores y expertos sobre los equipamientos empleados en deporte y las posibles modificaciones del reglamento es relevante (Ortega et al., 2012b; Ortega et al., 2008b; Tarodo et al., 2011; Toro et al., 2008; Vizcaíno et al., 2014), así como la opinión de los deportistas o árbitros (Coleclough, 2013; Francis \& Jones, 2014; Toro et al., 2015).

En este caso, se utilizó el sistema antivuelco para porterías de balonmano/futsal Tutigool. Es un novedoso sistema creado en España para la eliminación/ reducción de accidentes. Este sistema mejora incluso la normativa existente UNE-EN 749:2004/AC:2006 de "Equipos de campos de juego. Porterías de balonmano". Se utiliza un sistema antivuelco permanente, que garantiza la estabilidad de las porterías en cualquier parte del campo o pista y la seguridad de los usuarios en todo momento, favoreciendo la absorción de impactos durante el juego al no estar ancladas. Recientemente, ha sido motivo de dos estudios, los cuales han apoyado científicamente la modificación de la regla 1:2 en el reglamento oficial del balonmano (Baena-González et al., 2020a; Baena-González et al., 2020b); uno observando la influencia en el juego del sistema antivuelco Tutigool a través de metodología observacional y otro que expone la opinión de los participantes sobre dicho sistema antivuelco a través de un cuestionario, herramienta utilizada anteriormente en el estudio de Blanco et al. (2017).

Partiendo del cuestionario de Blanco et al. (2017), el cual está formado por 13 ítems de escala tipo Likert y que oscilan en un rango de (1) totalmente en desacuerdo a (7) totalmente de acuerdo, el objetivo de este estudio preliminar es validar las características psicométricas de una versión ampliada a 18 ítems del cuestionario. Con la validación de este instrumento en un estudio posterior se pretende tener una herramienta que permita conocer la opinión de los agentes implicados en los eventos deportivos (entrenadores, jugadores, árbitros, gestores, técnicos de las instalaciones deportivas, etc.), en el rendimiento del balon- mano/futsal con el sistema antivuelco de las porterías, equipamiento deportivo esencial para su práctica, así como causante de accidentes y muertes de deportistas, en su mayoría, menores de edad.

\section{Material y métodos}

\section{Participantes}

Panel de expertos. Para realizar el análisis de la validez de contenido del instrumento que se propone se consultó a un panel de 16 jueces expertos, compuesto por 4 entrenadores que poseen la titulación de entrenador nacional, 8 jugadores profesionales que han jugado al menos en dos divisiones diferentes en categoría absoluta y 4 árbitros de categoría nacional, todos ellos con más de 10 años de experiencia profesional en el balonmano español y tanto jugadores como entrenadores tienen experiencia internacional.

Muestra de jugadores, entrenadores y árbitros. Para obtener información acerca del nivel de comprensión y analizar la fiabilidad del cuestionario propuesto se realizó un estudio no probabilístico a partir de una muestra intencional formada por $\mathrm{n}=60$ participantes, compuesta por 51 jugadores de la categoría junior (85\%), 5 entrenadores (8.3\%) y 4 árbitros (6.7\%), de los cuales, el 3.3\% ( $\mathrm{n}=2)$ eran mujeres $(\mathrm{M}=27.5$; DT= $.07)$ y el $96.7 \%(n=58)$ eran hombres $(M=22.18$; $\mathrm{DT}=$ 8.86) durante el Torneo Internacional 4 Naciones celebrado en Santander (España) en 2019. Los jugadores y entrenadores participantes proceden de Alemania (30.4\%), España (37.5\%), Francia (3.6\%) y Portugal (28.6\%). Los jugadores participantes ocupan los siguientes puestos dentro del campo: portero (13.7\%), extremo (27.5\%), lateral (29.4\%), central (11.8\%), pivote $(17.6 \%)$. Cabe destacar que los participantes accedieron de forma voluntaria a aportar su contribución al desarrollo de la investigación.

\section{Instrumentos}

Instrumento de referencia. El instrumento de referencia fue creado por Blanco et al. (2017), cuestionario ad hoc formado por 13 ítems (Tabla 1) y cumplimentado por los participantes del Mundial Universitario 2016 celebrado en Antequera (España).

Entrevista estructurada. Al cuestionario de referencia se añadieron 5 ítems (resultando un total de 18 ítems) propuestos por los responsables de este estudio, sobre la base del análisis bibliográfico realizado y a partir de experiencias prácticas relacionadas con el uso del sistema antivuelco objeto de estudio, que corresponden a 
Tabla 1. Ítems existentes en el cuestionario de referencia.

\begin{tabular}{|c|c|c|c|c|c|c|c|}
\hline \multirow{2}{*}{$\frac{\text { Considero que ... }}{1 . . . \text { la propuesta de este tipo de portería es novedosa }}$} & \multicolumn{3}{|c|}{$\begin{array}{l}\text { Totalmente } \\
\text { de acuerdo }\end{array}$} & \multicolumn{4}{|c|}{$\begin{array}{c}\text { Totalmente } \\
\text { en desacuerdo }\end{array}$} \\
\hline & 1 & 2 & 3 & 4 & 5 & 6 & 7 \\
\hline 2. ... puede ser útil para aumentar la seguridad, al no estar anclada, durante el desarrollo del entrenamiento/juego & 1 & 2 & 3 & 4 & 5 & 6 & 7 \\
\hline $\begin{array}{l}\text { 3.... puede ser útil para aumentar la movilidad, ya que facilita el desplazamiento, durante el desarrollo del } \\
\text { entrenamiento/juego }\end{array}$ & 1 & 2 & 3 & 4 & 5 & 6 & 7 \\
\hline 4. ... puede aumentar la seguridad del portero & 1 & 2 & 3 & 4 & 5 & 6 & 7 \\
\hline 5. ... puede aumentar la seguridad del equipo en posición de ataque & 1 & 2 & 3 & 4 & 5 & 6 & 7 \\
\hline 6. ... puede aumentar la seguridad del equipo en posición de defensa & 1 & 2 & 3 & 4 & 5 & 6 & 7 \\
\hline 7. ... puede favorecer la continuidad del juego (a pesar de no estar fijada al suelo/pared) & 1 & 2 & 3 & 4 & 5 & 6 & 7 \\
\hline 8. ... la estructura es uniforme & 1 & 2 & 3 & 4 & 5 & 6 & 7 \\
\hline 9. ... la estructura es de difícil alteración & 1 & 2 & 3 & 4 & 5 & 6 & 7 \\
\hline 10. ... es conveniente para el juego/entrenamiento para escuelas deportivas municipales & 1 & 2 & 3 & 4 & 5 & 6 & 7 \\
\hline 11. ... es conveniente para el juego/entrenamiento para colegios & 1 & 2 & 3 & 4 & 5 & 6 & 7 \\
\hline 12. ... es conveniente para el juego del máximo nivel deportivo & 1 & 2 & 3 & 4 & 5 & 6 & 7 \\
\hline 13. Satisfacción general con la propuesta del sistema antivuelco & 1 & 2 & 3 & 4 & 5 & 6 & 7 \\
\hline
\end{tabular}

Tabla 2. Ítems nuevos propuestos.

\begin{tabular}{|c|c|c|}
\hline 1. Ya conocía este tipo de antivuelco & Sí & No \\
\hline 2. En mi país de origen existe este antivuelco & Sí & No \\
\hline 3. He tenido experiencia previa a este estudio/torneo con el sistema antivuelco & Sí & No \\
\hline 4. Su diseño me ha llamado la atención & Sí & No \\
\hline 5. ¿Cuántos accidentes/golpes ha presenciado contra porterías? & $\begin{array}{c}\text { Muchos }(>21) \\
\text { Bastantes }(16-20)\end{array}$ & $\begin{array}{c}\text { No muchos }(11-15) \\
\text { Pocos }(6-10) \\
\text { Muy pocos }(1-5) \\
\text { Ninguno }\end{array}$ \\
\hline
\end{tabular}

dos bloques (Tabla 2): el primero de ellos, con 4 ítems de respuesta dicotómica sí/no, referentes al conocimiento y diseño del sistema antivuelco, y el segundo, con un ítem referente a la cantidad de accidentes/golpes presenciados contra porterías, de respuesta tipo escala nominal (muchos, bastantes, no muchos, pocos, muy pocos, ninguno).

Para alcanzar niveles óptimos de validez de contenido del instrumento propuesto se utilizó la técnica de jueces expertos. De este modo, se diseñó una entrevista estructurada y un instrumento para la evaluación cualitativa de los ítems existentes y los 5 nuevos propuestos (Tabla 2).

Evaluación de los ítems por parte de los expertos. Cada ítem se valora utilizando una escala cualitativa ordinal tipo Likert con 5 categorías (1: nada de acuerdo; 5: totalmente de acuerdo). Los ítems con valoración media inferior a 3 puntos (nivel neutral) en una escala 1-5 serían necesariamente modificados o eliminados del cuestionario. Los 18 ítems recibieron una valoración media superior a 3 puntos por parte de los expertos.

Instrumento definitivo. El número de ítems del cuestionario definitivo fue de 18 , es decir, se añadieron los 5 nuevos ítems propuestos al cuestionario original. Se ha considerado por parte de los investigadores y expertos que cada ítem aporta información relevante sobre el sistema antivuelco y que la extensión del cuestionario modificado es adecuada al contener un número mínimo de preguntas necesarias para lograr el objetivo de la investigación.

\section{Procedimiento}

En una primera fase, los investigadores revisaron la estructura y contenido del instrumento aportado por Blanco et al. (2017). Se consideró que era conveniente mantener los 13 ítems iniciales sin cambios y añadir 5 ítems con redacción propia para incluir aspectos no considerados en el instrumento base y que pueden ser esenciales en el estudio de percepción del sistema antivuelco.

La segunda fase tenía por objetivo someter el instrumento propuesto a consideración de jueces expertos para analizar la validez de contenido y realizar modificaciones oportunas antes de su aplicación práctica, teniendo en cuenta aportaciones de tipo cualitativo sobre la pertinencia de los ítems, o sobre la forma de redactar cada uno de ellos. Se contactó individualmente a cada miembro del panel de expertos para explicar los objetivos de la investigación y aspectos relacionados con la evaluación del instrumento. Se facilitó a 
cada juez vía correo electrónico una copia del instrumento a validar con la totalidad de ítems originales y propuestos. Se concertaron entrevistas estructuradas independientes. La participación de los expertos fue voluntaria y anónima.

En la tercera fase de la investigación se procesaron los resultados de las entrevistas realizadas a cada uno de los 16 expertos. Se procesaron las respuestas relacionadas con la evaluación cualitativa de cada ítem y se tuvieron en cuenta sus opiniones para modificar y, en definitiva, mejorar la calidad del cuestionario.

En la cuarta fase se aplicó el instrumento validado previamente por expertos a una muestra formada por 60 participantes (jugadores, entrenadores y árbitros). Durante el proceso de cumplimentación de los cuestionarios, el investigador principal resolvió dudas generales que pudieran surgir y observó el nivel de comprensión que tenían los participantes acerca de los ítems que conformaban el instrumento aplicado. La recogida de los instrumentos se realizó de forma individual y se hicieron las revisiones pertinentes para garantizar el mayor número de respuestas válidas posibles asociadas a la comprensión del instrumento.

En la quinta fase del estudio se procedió a la construcción de la base de datos y al análisis estadístico de resultados para verificar la comprensión del instrumento por parte de los participantes, proponer nuevos cambios de redacción si fuera necesario y obtener conclusiones preliminares de forma general acerca de la validez y fiabilidad del cuestionario.

\section{Análisis estadístico}

Los datos procedentes de la entrevista estructurada aplicada al panel de expertos (evaluación de ítems mediante escala Likert) y de la aplicación del cuestionario propuesto a los 60 participantes en el estudio cuantitativo (jugadores, entrenadores y árbitros) se estructuraron en dos bases de datos construidas sobre Microsoft Excel 2016. El procesamiento estadístico de datos se realizó mediante el programa IBM SPSS Statistics, versión 23 para Windows. Se han aplicado métodos de análisis descriptivo de variables, construcción de tablas de frecuencias y de contingencia, pruebas de normalidad de Kolmogorov-Smirnov, cálculo del Coeficiente de Fiabilidad a de Cronbach + ANOVA con prueba de Friedman y análisis factorial exploratorio (AFE) (método de componentes principales y rotación Varimax). En cuanto a los ítems, se realizaron estudios de correlación bivariada no paramétrica ( $\rho$ de Spearman). Para la adecuación de muestral se utilizó la medida KMO (Kaiser-Meyer-Olkin) de adecuación muestral y prueba de esfericidad de Bartlett.

\section{Resultados}

La validez de contenido del cuestionario se comprueba a través de los resultados del estudio de expertos realizado. Las entrevistas realizadas han permitido obtener opiniones y sugerencias de los expertos en cuanto a diseño, estructura, redacción, idoneidad, comprensión y adecuación para describir las dimensiones del problema bajo estudio (percepción en el juego y el rendimiento en la utilización del equipamiento deportivo: sistema antivuelco de las porterías). Las evaluaciones de los ítems que conforman el instrumento han sido favorables en general: $(4.55 \pm .623)$ puntos, en una escala 1-5: ni de desacuerdo ni en desacuerdo (6,9\%), de acuerdo (31.5\%), totalmente de acuerdo $(61,5 \%)$.

Se ha aplicado el cuestionario propuesto a una muestra de 60 participantes para comprobar el nivel de comprensión de los ítems y con el objetivo de evaluar el funcionamiento del instrumento, explorar características internas y averiguar si las relaciones entre las variables consideradas definen una estructura dimensional coherente, que se mantenga invariante y que pueda aplicarse para la interpretación de los resultados en universos diferentes (Baena-González et al., 2020a). Los resultados indican que el $18.3 \%$ de los participantes ya conocía el sistema antivuelco en el momento del estudio. Todos ellos son jugadores. Del mismo modo el $26.7 \%$ de los jugadores afirma que este sistema antivuelco existe en sus países de origen. $\mathrm{Ni}$ los entrenadores ni los árbitros encuestados conocían el sistema antivuelco Tutigool y ninguno de los participantes había tenido experiencia previa con el mismo. El diseño ha llamado la atención al $48.3 \%$ de los participantes, fundamentalmente a jugadores (49\%). Tabla 3.

El instrumento que se aporta permite determinar los niveles de acuerdo de jugadores, entrenadores y árbitros acerca de la novedad, seguridad, continuidad del juego, estructura, conveniencia y opinión general del sistema propuesto, en una escala de evaluación 1-7, y realizar estudios de correlaciones, comparaciones entre grupos, etc. Los ítems de evaluación que se han incluido presentan índices de homogeneidad estadísticamente significativos, lo que indica que cada uno de ellos se correlaciona significativamente con la suma de los restantes ítems. Tabla 4.

La pregunta relacionada con accidentes/golpes presenciados ha sido respondida por el $100 \%$ de los participantes. La escala de medición utilizada permite realizar cruces de variables y análisis de correlaciones con los restantes ítems que conforman el instrumento. Se observan diferencias significativas en los niveles 
Tabla 3. Conocimiento, experiencia y diseño del sistema antivuelco Tutigool.

\begin{tabular}{|c|c|c|c|c|}
\hline & Jugadores & Entrenadores & Árbitros & Total \\
\hline \multirow{2}{*}{ Conocía este sistema antivuelco } & $11 / 51$ & 0 & 0 & $11 / 60$ \\
\hline & $21.5 \%$ & $0 \%$ & $0 \%$ & $18.3 \%$ \\
\hline \multirow{2}{*}{ Existe en mi país de origen } & $16 / 51$ & 0 & 0 & $16 / 60$ \\
\hline & $31.3 \%$ & $0 \%$ & $0 \%$ & $26.7 \%$ \\
\hline \multirow{2}{*}{ Diseño le ha llamado la atención } & $25 / 51$ & $3 / 5$ & $1 / 4$ & $29 / 60$ \\
\hline & $49 \%$ & $60 \%$ & $25 \%$ & $48.3 \%$ \\
\hline
\end{tabular}

Tabla 4. Parámetros descriptivos e índice de homogeneidad de los ítems

\begin{tabular}{lccccc}
\hline \multirow{2}{*}{ Variables } & Jugadores & Entrenadores & Árbitros & Total & Índice de \\
\cline { 2 - 4 } homogeneidad
\end{tabular}

** Correlaciones significativas ( $\rho$ de Spearman), $p<.01$

de respuestas entre jugadores, entrenadores y árbitros $(\mathrm{p}<$.05). El número de golpes presenciados en el juego influye significativamente sobre las opiniones que tienen los participantes acerca de este sistema antivuelco en cuanto a la novedad, seguridad, facilidad para la continuidad de juego, conveniencia para escolares y para lograr el máximo rendimiento deportivo $(p<.05)$. Tabla 5.

Las pruebas de normalidad de Kolmogorov-Smirnov indican que en general los datos correspondientes a cada variable se alejan significativamente de la distribución normal ( $p<.05)$. Se seleccionan pruebas estadísticas no paramétricas y coeficientes de correlación no paramétricos para el procesamiento de los mismos.

Se obtiene una medida de adecuación muestral KMO de .764 en una escala 0-1, lo cual indica la existencia de correlaciones significativas entre las variables consideradas. La prueba de esfericidad de Bartlett $\left(X^{2}=509.4, \mathrm{gl}=105, \mathrm{p}=.000\right)$. Estos resultados demuestran la presencia de correlaciones significativas entre las variables que integran el instrumento. El análisis factorial exploratorio demuestra que existe una estructura factorial definida formada por tres factores que explican el $61.58 \%$ de la varianza de los datos. Se ha considerado un número mínimo de tres ítems en cada factor y una carga factorial mínima de .40 , según recomendaciones de la literatura (Mavrou, 2015). Tabla 6.

Se obtiene un coeficiente de fiabilidad a de Cronbach igual .860 en una escala 0-1 sobre la base de 18 ítems que presentan varianza significativamente diferente de cero, que indica una consistencia interna adecuada de los ítems. La prueba $X^{2}$ de Friedman permite rechazar la hipótesis de que no existen diferencias significativas entre las medianas de las distribuciones de probabilidad de las variables asociadas a cada ítem ( $\mathrm{p}<$ .05), lo cual indica que de forma individual cada pregunta incluida en el instrumento aporta información relevante. Tabla 7 .

\section{Discusión}

El objetivo principal de este trabajo es realizar un estudio preliminar sobre la ampliación y validación del cuestionario ad-hoc de Blanco et al. (2017) a 18 ítems que miden la satisfacción de los agentes implicados en los eventos deportivos (entrenadores, jugadores, 
Tabla 5. Tabla de contingencia. Accidentes/golpes presenciados (M, B, NM, P, MP, N).

\begin{tabular}{|c|c|c|c|c|c|c|}
\hline & & & Jugador/a & Entrenador/a & Árbitro/a & Total \\
\hline \multirow{12}{*}{$\begin{array}{l}\text { Golpes/accidentes } \\
\text { presenciados } \\
(\mathrm{M}, \mathrm{B}, \mathrm{NM}, \mathrm{P}, \mathrm{MP}, \mathrm{N})\end{array}$} & \multirow{2}{*}{ Muchos (>21) } & Recuento & 1 & 1 & 0 & 2 \\
\hline & & $\%$ del total & $1.70 \%$ & $1.70 \%$ & $.00 \%$ & $3.30 \%$ \\
\hline & \multirow{2}{*}{ Bastantes (16-20) } & Recuento & 11 & 0 & 1 & 12 \\
\hline & & $\%$ del total & $18.30 \%$ & $.00 \%$ & $1.70 \%$ & $20 \%$ \\
\hline & \multirow{2}{*}{ No muchos (11-15) } & Recuento & 6 & 1 & 3 & 10 \\
\hline & & $\%$ del total & $10 \%$ & $1.70 \%$ & $5 \%$ & $16.70 \%$ \\
\hline & \multirow{2}{*}{ Pocos $(6-10)$} & Recuento & 10 & 0 & 0 & 10 \\
\hline & & $\%$ del total & $16.70 \%$ & $.00 \%$ & $.00 \%$ & $16.70 \%$ \\
\hline & \multirow{2}{*}{ Muy pocos (1-5) } & Recuento & 12 & 2 & 0 & 14 \\
\hline & & $\%$ del total & $20 \%$ & $3.30 \%$ & $.00 \%$ & $23.30 \%$ \\
\hline & \multirow{2}{*}{ Ninguno } & Recuento & 11 & 1 & 0 & 12 \\
\hline & & $\%$ del total & $18.30 \%$ & $1.70 \%$ & $.00 \%$ & $20 \%$ \\
\hline \multirow{2}{*}{ Total } & & Recuento & 51 & 5 & 4 & 60 \\
\hline & & $\%$ del total & $85 \%$ & $8.30 \%$ & $6.70 \%$ & $100 \%$ \\
\hline
\end{tabular}

$\mathrm{X} 2$ de Pearson $=18.793, \mathrm{gl}=10, \mathrm{p}$ (asintótica bilateral $)=.043$

Tabla 6. Matriz de componentes rotados.

\begin{tabular}{|c|c|c|c|}
\hline & \multicolumn{3}{|c|}{ Componente } \\
\hline & 1 & 2 & 3 \\
\hline Puede ser útil para aumentar la movilidad & .912 & & \\
\hline Puede ser útil para mejorar la seguridad al no estar anclada & .817 & & \\
\hline Puede aumentar la seguridad del portero & .790 & & \\
\hline La propuesta de este tipo de portería es novedosa & .570 & & \\
\hline Puede favorecer la continuidad del juego a pesar de no estar anclada & .506 & & \\
\hline Golpes/accidentes presenciados & -.476 & & \\
\hline Puede aumentar la seguridad del equipo en posición de defensa & & -.792 & \\
\hline Puede aumentar la seguridad del equipo en posición de ataque & & -.752 & \\
\hline El diseño le ha llamado la atención & & .543 & \\
\hline La estructura es uniforme & & & .788 \\
\hline La estructura es de difícil alteración & & & .775 \\
\hline Es conveniente para escuelas deportivas municipales & & & .650 \\
\hline Es conveniente para escolares & & & .600 \\
\hline Es conveniente para máximo nivel deportivo & & & .486 \\
\hline
\end{tabular}

Tabla 7. ANOVA con prueba de Friedman.

\begin{tabular}{llcccc}
\hline & & Suma de cuadrados & Gl & Media cuadrática & Chi-cuadrado de Friedman \\
\hline Inter sujetos & & 792.642 & 58 & 13.666 & Sig \\
\hline Intra sujetos & Entre elementos & $2743.998^{\mathrm{a}}$ & 16 & 171.500 & 573.336 \\
& Residuo & 1774.002 & 928 & 1.912 & .000 \\
& Total & 4518.000 & 944 & 4.786 & 5.300 \\
\hline Total & 5310.642 & 1002 & \\
\hline
\end{tabular}

Media global $=3.76$. . Coeficiente de concordancia de $W=.517$. 
gestores, técnicos de las instalaciones deportivas, etc.) con el rendimiento en el balonmano/futsal del Sistema Antivuelco de las porterías.

En este sentido y según los resultados obtenidos, se refuerza la posible utilización del cuestionario como instrumento de investigación, siendo una excelente herramienta para investigar sobre el sistema antivuelco de las porterías.

El proceso de validación, en una primera fase general para evaluar la presentación, instrucciones, dificultad, orden y extensión del cuestionario, resultó muy adecuado para cuantificar aspectos cualitativos de la herramienta elaborada (Sierra, 2001).

Las aportaciones realizadas por los jueces-expertos para la validación de contenido son indispensables en el desarrollo de un instrumento (Bulger \& Housner, 2007), además de considerarse un elemento esencial para proporcionar evidencias teóricas de validez ( $\mathrm{Ru}-$ bio et al., 2003). En este sentido, permitieron ajustar tanto el contenido como la redacción de las preguntas (Ortega et al., 2008a). Del mismo modo, el grupo multidisciplinar de 16 sujetos (expertos) es un número aceptable para la validación del instrumento según estudios anteriores (Juan-Llamas, 2015; Ortega et al., 2008c; Robles et al., 2016).

El método busca el consenso de expertos para determinar si el contenido de los ítems es adecuado y si el instrumento sirve para registrar con rigurosidad lo que se pretende analizar. Por ello, los jueces ofrecieron aportaciones cuantitativas y cualitativas que permitieron mejorar el instrumento, obteniendo valores muy positivos en todas las dimensiones y sus categorías de valoración: pertinencia de las preguntas y sus categorías de respuesta en relación al objeto de estudio, claridad y comprensión en la redacción, estructura y orden adecuado de las dimensiones y preguntas, y criterio de exhaustividad. La valoración de los expertos sobre los ítems que conforman el instrumento ha sido favorable en general (4,55 sobre 5$)$.

Los ítems de evaluación presentan índices de homogeneidad estadísticamente significativos, lo que indica que cada uno de ellos se correlaciona significativamente con las sumas de los restantes (González, 2007). Del mismo modo, se obtiene un coeficiente de fiabilidad a de Cronbach igual .860 en una escala 0 -1, sobre la base de 18 ítems que presentan varianza significativamente diferente de cero, que indica una consistencia interna adecuada de los ítems, es decir, es confiable (Gálvez \& Morales, 2014; Latorre \& Pantoja, 2013; SánchezPato et al., 2016).

Para la comprensión y evaluación del funcionamiento de los ítems del instrumento se ha realizado un estudio cuya muestra es de 60 participantes (Baena-González et al., 2020a), y las variables consideradas definen una estructura dimensional coherente, cuyos resultados pueden aplicarse a futuras investigaciones sobre el sistema antivuelco de las porterías de balonmano o futsal. Es importante destacar lo expuesto por Ochoa (2015), quien define el muestreo por conveniencia como una técnica comúnmente utilizada que se caracteriza por su fácil accesibilidad; los individuos se seleccionan sin usar ningún criterio estadístico. Esto a su vez implica fácil operatividad y menores costos.

Del mismo modo, cada pregunta del cuestionario de forma individual incluida en el instrumento aporta información relevante, así lo indica la $X^{2}$ de Friedman, la cual permite rechazar la hipótesis de que no existen diferencias significativas entre las medianas de las distribuciones de probabilidad de las variables asociadas a cada ítem ( $\mathrm{p}<.05$ ), prueba utilizada en varios estudios (Flores et al., 2016; Taboada \& Ferrer, 2019).

\section{Conclusiones}

El estudio preliminar realizado ha pretendido mostrar que la metodología aplicada es apropiada; así lo refleja la escala analizada, pues parece indicar una adecuada fiabilidad a través del alfa de Cronbach con un valor de .860 en una escala 0-1. Además, sería recomendable aumentar la muestra del estudio y realizar un análisis factorial confirmatorio para constatar los criterios de fiabilidad y validez, tanto convergente como discriminante.

Desde el punto de vista de la seguridad en los eventos deportivos, una vez analizados los procesos de validez y fiabilidad preliminares del cuestionario, a pesar de las limitaciones indicadas se considera que el instrumento diseñado podría aplicarse para conocer la opinión de los agentes deportivos acerca de la utilización del sistema antivuelco Tutigool en las porterías de balonmano/futsal.

\section{Aplicaciones prácticas}

Con el cuestionario utilizado en este estudio preliminar, los investigadores, los responsables de las instalaciones deportivas y los organizadores de eventos deportivos podrían contar con un instrumento útil y práctico para estudiar un equipamiento deportivo que provoca lesiones y muertes en los deportistas.

\section{Limitaciones}

Algunas de las limitaciones del presente estudio son las posibles malas interpretaciones del idioma, pues 
solo se hizo el cuestionario en inglés y español. Igualmente, debe realizarse el análisis factorial confirmatorio $(\mathrm{AFC})$ para la correcta validación del instrumento, y para ello se debe aumentar el tamaño de la muestra, pues un AFC con la muestra actual es deficitaria y la literatura no lo considera adecuado.

\section{Futuros estudios}

Futuras investigaciones podrán utilizar esta herramienta con otros universos muestrales y como guía para crear otros cuestionarios que permitan estudiar diferentes equipamientos deportivos.

\section{Conflicto de intereses}

Los autores no declaran conflicto de intereses.

\section{Agradecimientos}

Los autores agradecen a D. Antonio González Cánovas la cesión del uso Sistema Antivuelco Tutigool, a la Real Federación Española de Balonmano, a la Federación Alemana de Balonmano y a la Federación Internacional de Balonmano. Los resultados obtenidos en esta investigación han contribuido a la adopción del artículo 1:2 del reglamento oficial del balonmano.

\section{BIBLIOGRAFÍA}

Angulo, A. G., Egido, J. M., Angulo, F. J., \& Toro, E. O. (2019). Revisión de los reglamentos de balonmano en categorías de formación en España. E-balonmano. Com: Revista De Ciencias Del Deporte, 15(1), 9-22. http:// www.e-balonmano.com/ojs/index.php/revista/article/view/432

Andrew, D. P., Chow, J. W., Knudson, D. V., \& Tillman, M. D. (2003). Effect of ball size on player reaction and racket acceleration during the tennis volley. Journal of Science and Medicine in Sport, 6(1), 102-112. https://doi.org/10.1016/S1440-2440(03)80013-0

Baena-González, R., García-Tascón, M., Chavarría-Ortiz, C., MartínezMartín, I., \& Gallardo, A. M. (2020a). Opinion of handball players, trainers and referees using the handball/futsal goalpost anti-tip system "Tutigool" for a safe game: 2019 Four Nations International Handball Junior Tournament. Journal of Physical Education and Sport, 20(4), 1695-1705. https://doi.org/10.7752/jpes.2020.04230

Baena-González, R., Lozano, D., Gallardo, A. M., Chavarría-Ortiz, C., \& García-Tascón, M. (2020b). Influence of the handball goal anti-tip system through the game actions observation method: 2019 Four Nations International Handball Junior Tournament. International Journal of Performance Analysis in Sport, 20(3), 357-372. https://doi.org/1 0.1080/24748668.2020.1749967

Babí J., Inglé, E., Cumellas, L., Farías, E. I., Seguí, J., \& Labrador, V. (2018). El perfil de los corredores y su propensión al accidente deportivo. Revista Internacional de Medicina y Ciencias de la Actividad Física y Del Deporte, 18(72), 723. https://doi.org/10.15366/rimcafd2018.72.009

Blanco, D., Diaz-Cabrera, J. A., Torres-Pinazo, J., Carmona-Álamos, L. C., \& García-Tascón , M. (2017). Satisfacción de los usuarios con el Sistema Antivuelco para Porterías de balonmano/fútbol Sala Tutigool: Campeonato Mundial Universitario Balonmano 2016. Estudio piloto. En J. Corral, \& C. Gómez-González, El uso de datos en la Economía del Deporte. Mirando hacia el futuro (pp. 308-311). Ediciones de la Universidad de Castilla-La Mancha.

Bulger, S. M., \& Housner, L. D. (2007). Modified Delphi Investigation of Exercise Science in Physical Education Teacher Education, Journal of Teaching in Physical Education, 26(1), 57-80. https://journals.humankinetics.com/view/journals/jtpe/26/1/article-p57.xml

Cherington, M. (2001). Lightning Injuries in Sports. Sports Medicine, 31(4), 301-308. https://doi.org/10.2165/00007256-200131040-00004

Coleclough, J. (2013). Soccer coaches' and referees' perceptions of tackle incidents with respect to the laws of the game. International Journal of Performance Analysis in Sport, 13(2), 553-566. https://doi.org/10.108 0/24748668.2013.11868669

Del Campo, V. L., \& Sánchez, R. S. (2016). Análisis y evaluación de la seguridad de instalaciones y equipamientos deportivos escolares en la ciudad de Mérida (Extremadura). RETOS. Nuevas Tendencias en Educación Física, Deporte y Recreación, 29, 66-71. https://recyt.fecyt.es/ index. php/retos/article/view/34364/21776

Dosseville, F. E. (2007). Influence of ball type on home advantage in French professional soccer. Perceptual and Motor Skills, 104(2), 347351. https://doi.org/10.2466/PMS.104.2.347-351
European Association for Injury Prevention and Safety Promotion. (2010). Eurosafe. www.euro.safe.eu.com/publication/policy-briefing

Flores, K., López, M. C., \& Rodríguez, M. A. (2016). Evaluación de componentes de los cursos en línea desde la perspectiva del estudiante. Revista Electrónica de Investigación Educativa, 18(1), 23-38. http:// redie.uabc.mx/redie/article/view/474

Francis, J., \& Jones, G. (2014). Elite Rugby Union Players Perceptions of Performance Analysis. International Journal of Performance Analysis in Sport, 14(1), 188-207. https://doi.org/10.1080/24748668.2014.1 1868714

Gálvez-Ruiz, P., \& Morales-Sánchez, V. (2015). Desarrollo y validación del cuestionario para la evaluación de la calidad percibida en servicios deportivos. Cultura, Ciencia y Deporte, 28 (10), 55-66. https://doi. org/10.12800/ccd.v10i28.515

García-Tascón, M., Gallardo, A. M., Blanco, D., Martínez-López, Á. J., \& Márquez, I. (2014). Análisis del cumplimiento de la seguridad de los equipamientos deportivos del municipio de Sevilla (España). Cultura, Ciencia y Deporte, 9(26), 129-138. http://dx.doi.org/10.12800/ccd. v9i26.431

García-Tascón, M. (2018a). La Universidad Pablo de Olavide (Sevilla) ha apostado por el avance en la investigación en la seguridad deportiva. news.mondoiberica. https://news.mondoiberica.com.es/universidadpablo-olavide-sevilla-apuesta-investigacion-seguridad-deportiva/

García-Tascón, M. (2018b). Más allá de la responsabilidad jurídica del gestor. Deporcam, 40,6-7. https://www.circulodegestores.com/recursos/documentos/send/3-revista-deporcam/30-revistadeporcam-n\%C2\%BA40

Gavilán, F. (2011). Un total de 16 menores han muerto por la caída de una portería o una canasta en España. IDEAL. https://www.ideal.es/granada/20110302/local/almeria/totalmenores-muertocaida-201103012247.html

Giatsis, G., \& Tzetzis, G. (2003). Comparison of performance for winning and losing beach volleyball teams on different court dimensions. International Journal of Performance Analysis in Sport, 3(1), 65-74. https://doi.org/10.1080/24748668.2003.11868276

González, F. M. (2007). Instrumentos de evaluación psicológica. Louisiana, Habana: Editorial Ciencias Médicas 248 (247), 234. https://scholar. google.com/scholar_lookup?hl=en\&publication_year=2007\&pages= 234\&issue $=247$ \&author=F.+M.+Gonz\%C3\%A1lez+Llaneza\&author= M.+Mart\%C3\%ADn+Carbonell\&title=Instrumentos+de+evaluaci\% C3\%B3n+psicol\%C3\%B3gica

Heino, R. (2000). New Sports: What is So Punk about Snowboarding? Journal of Sport and Social Issues, 24(2), 176-191. https://doi.org/10. 1177/0193723500242005

Hernández-Sánchez, J. H., \& García-Tascón, M. (2016). Revisión de estudios e investigaciones sobre la prevención de accidentes y lesiones en educación física: Propuestas y medidas para minimizar o evitar riesgos. EmásF: Revista Digital de Educación Física, 43, 25-52. https:// emasf.webcindario.com/Revision_de_estudios_e_investigaciones_ sobre_la_prevencion_de_accidentes_y_lesiones_en_EF.pdf 
IHF (2019). ihf.info. https://www.ihf.info/regulations-documents/361? selected=Rules\%20of\%20the\%20Game (accessed on 25 April 2020).

Llamas, C. (2015). Diseño y validación de un cuestionario sobre la forma de trabajo de los instructores de clases colectivas. Retos, Nuevas tendencias en Educación, Física, Deporte y Recreación, 27, 19-23. https:// eprints.ucm.es/46052/

Katthage, J., \& Thieme-Hack, M. (2013). Sportplatz als Abenteuerspielplatz. Stadt + Grün, 9, 49-54. https://stadtundgruen.de/artikel/ sportplatz-als-abenteuerspielplatz-4264

Kisser, R., \& Bauer, R. (2012). The burden of sports injuries in the European Union. Injury Prevention, 16. https://doi.org/10.1136/ ip.2010.029215.752

Krauss, M. D. (2004). Equipment innovations and rules changes in sports. Current Sports Medicine Reports 3(5), 272-276. https://doi. org/10.1007/s11932-004-0053-6

Laferrier, J. Z., Rice, I., Pearlman, J., Michelle, L., Sporner, M., Cooper, R. M., Liu, H., \& Cooper, R. A., (2012). Technology to Inprove Sport Performance in Wheelchair Spots, Sports Technology, 5(1-2), 4-19. https://doi.org/10.1080/19346182.2012.663531

Latorre, P. A. (2008). Metodología para el análisis y evaluación de la seguridad de los espacios y equipamientos deportivos escolares. Revista Apunts Educación Física y Deportes, 93, 62-70.

Latorre, P. A., Mejía, J. A., Gallego, M., Muñoz, A., Santos, M. A., \& Adell, M. (2012). Análisis de la seguridad de las instalaciones deportivas de las sedes de los juegos deportivos provinciales de jaén. Journal of Sport \& Health Research, 4(1), 57-66. http://www.journalshr.com/papers/ Vol\%204_N\%201/V04_1_6.pdf

Latorre, P.A., \& Pantoja, A. (2013). Diseño y validación de un cuestionario de propensión al accidente deportivo. Cuadernos de Psicología del Deporte, 13(1), 51-62. https://doi.org/10.4321/s1578-84232013000100006

Ley 53/2002, de Medidas Fiscales, Administrativas y del Orden Social. 31 de diciembre de 2002. Boletín Oficial del Estado. No.13 https://www.boe.es/eli/es/1/2002/12/30/53/con

Magaz-González, A. M., \& Fanjul-Suárez, J. L. (2012). Organización de eventos deportivos y gestión de proyectos: factores, fases y áreas. $R e-$ vista Internacional de Medicina y Ciencias de la Actividad Física y el Deporte, 12(45), 138-169. http://cdeporte.rediris.es/revista/revista45/ artorganizacion209.htm

Macan J, Bundalo-Vrbanac D., \& Romić, G. (2006). Effects of the new karate rules on the incidence and distribution of injuries. British Journal of Sports Medicine, 40(4), 326-330. http://dx.doi.org/10.1136/bjsm. 2005.022459

Mavrou, I. (2015). Análisis factorial exploratorio: cuestiones conceptuales y metodológicas. Revista Nebrija de lingüística aplicada a la enseñanza de las lenguas, 19, 71-80. https://revistas.nebrija.com/revista-linguistica/issue/view/25/numero\%2019

Montalvo, J., Felipe, J. L., Gallardo, L., Burillo, P., \& García-Tascón, M. (2010). Las instalaciones deportivas escolares a examen: Una evaluación de los institutos de educación secundaria de Ciudad Real. Retos. Nuevas tendencias en Educación, Física, Deporte y Recreación, 17, 59-61. https://recyt.fecyt.es/index.php/retos/article/view/34679/18787

Ochoa, C. (2015). Non-probabilistic sampling: convenience sampling. Obtained from non-probabilistic sampling: convenience sampling: https:// www.netquest.com/blog/es/blog/es/muestreo-por-conveniencia

O'Donoghue, G. P. (2012). The effect of rule changes in World Series Netball: A simulation study. International Journal of Performance Analysis in Sport, 12(1), 90-100. https://doi.org/10.1080/24748668.201 2.11868585

Ortega, E., Alarcón, F., \& Piñar, M. J. (2012a). Modificaciones reglamentarias en baloncesto de formación: Un nuevo equipamiento, una nueva perspectiva. En A. Antúnez, S. Ibáñez (coords.), El camino hacia la excelencia en baloncesto (pp.135-159). Wanceulen.https://books. google.de/books?hl=es\&lr=\&id=ai8ACwAAQBAJ\&oi=fnd\&pg=PA13 $5 \& \mathrm{dq}=$ nuevo + deporte\&ots=AdanwenUT-\&sig=I0OXDe_OXoFeaJV WL0ijjKW0r50\#v=onepage\&q=nuevo\%20deporte\&f=false

Ortega, E., Calderón, A., Palao, J. M., \& Puigcerver, C. (2008a). Diseño y validación de un cuestionario para evaluar la actitud percibida del profesor en clase y de un cuestionario para evaluar los contenidos actitudinales de los alumnos durante las clases de educación física en secundaria. Retos: Nuevas Tendencias en Educación Física, Deporte y Recreación, 14, 22-29. https://www.redalyc.org/articulo. oa?id=3457/345732279004
Ortega, E., Castro, J. M., \& Laporta, F. (2008b). Análisis de la opinión de entrenadores sobre la adecuación del reglamento a las necesidades de la categoría infantil en baloncesto. Cultura, Ciencia y Deporte, 5,76. https://issuu.com/ameba-entrenadores/docs/practicum

Ortega, E., Jiménez, J. M., Palao, J. M., \& Sainz, P. (2008c). Diseño y validación de un cuestionario para valorar las preferencias y satisfacciones en jóvenes jugadoras de baloncesto. Cuadernos de Psicología del Deporte, 8(2), 39-58. https://revistas.um.es/cpd/article/view/ $54281 / 52301$

Ortega, E., Piñar, M. I., Salado, J., Palao, J. M., \& Gómez, M. A. (2012b) Análisis del reglamento de la competición infantil en baloncesto (Analysis of the competition rules of children in basketball). RICYDE. Revista Internacional de Ciencias Del Deporte, 29(8), 142-150. http:// doi.org/10.5232/ricyde2012.02803

Ratten V. (2019). Sports, technology and innovation: assessing cultural and social factors. Springer Nature Switzerland AG. https://doi. org/10.1007/978-3-319-75046-0

Robles, A., Robles, J., Giménez, F. J., \& Abad, M. T. (en prensa). Validación de una entrevista para estudiar el proceso formativo de judokas de élite. Revista Internacional de Medicina y Ciencias de la Actividad Física y el Deporte, 16(64), 723-738. https://www.redalyc.org/articulo. oa?id=542/54248818007

Ronglan, L. T., \& Grydeland, J. (2006). The effects of changing the rules and reducing the court dimension on the relative strengths between game actions in top international beach volleyball. International Journal of Performance Analysis in Sport, 6(1), 1-12. https://doi.org/10.108 0/24748668.2006.11868351

Rubio, D. M., Berg-Weger, M., LCSW, Tebb S. S., Lee E. S., \& Rauch, S (2003) Objectifying content validity: Conducting a content validity study in social work research. Social Work Research, 27(2), 94104, https://doi.org/10.1093/swr/27.2.94

Sánchez-Pato, A., Calderón, A., Arias-Estero, J. L., García-Roca, J. A., Bada, J., Meroño, L., Isidori, E., Bruton, J., Decelis, A., Koustelios, A., Mallia, O., Fazio, A., Radcliffe, J., \& Sedgwick, M. (2016). Diseño y validación del cuestionario de percepción de los estudiantes universitarios-deportistas de alto nivel sobre la carrera dual (ESTPORT). Cultura, Ciencia y Deporte, 11(32), 127-147. http://dx.doi.org/10.12800/ccd.v11i32.713

Sierra, B. R. (2001). Técnicas de Investigación Social: teoría y ejercicios (Decimocuarta ed.). Paraninfo

Taboada, N., \& Ferrer, R. L. (2019). Validación de un cuestionario sobre factores de riesgo para defectos congénitos. Revista Cubana de Investigaciones Biomédicas, 38(4), e311. http://scielo.sld.cu/scielo.php?script=sci_ arttext\&pid=S0864-03002019000400011\&lng=es\&tlng=es

Tarodo, J. S., Belmonte, M. B., Toro, E. O., \& Ruano, M. G. (2011). Opinión de los entrenadores sobre distribución de contenidos técnico-tácticos y pedagógicos en distintas categorías de baloncesto de formación. Cuadernos de Psicología del Deporte, 11(2), 51-62. https:// revistas.um.es/cpd/article/view/132931

Toro, E. O., Egido, J. J., Andrés, J. P., \& de Barranda, P. S. (2008). Diseño y validación de un cuestionario para valorar las preferencias y satisfacciones en jóvenes jugadores de baloncesto. Cuadernos de Psicología del Deporte, 8(2), 39-58. https://revistas.um.es/cpd/article/view/54281

Toro, E. O., Angulo, A. G., \& Moreno, R. M. (2015). Modificación del reglamento de balonmano en etapas de formación según la opinión de los jugadores. AGON, 5(1), 27-34. https://dialnet.unirioja.es/servlet/ articulo? codigo $=6706990$

Vizcaíno, C., Sáenz-López, P., \& Rebollo, J. A. (2013). Revisión de los reglamentos de minibasket en las comunidades autónomas de España. e-balonmano.com: Revista de Ciencias del Deporte, 9(3), 173-192. www.e-balonmano.com/ojs/index.php/revista/article/view/140/148

Vizcaíno, C. D., Sáenz-López, P. B., Rebollo, G. J., \& Conde, C. G. (2014) Opinión de entrenadores, árbitros y expertos sobre la enseñanza del minibasket desde una perspectiva cualitativa. RETOS. Nuevas Tendencias en Educación Física, Deporte y Recreación, 25, 9-12. http://www. redalyc.org/articulo.oa?id=345732291002

Williams, J., Hughes, M., \& O'Donoghue, P. (2005). The effect of rule changes on match and ball in play time in rugby union. International Journal of Performance Analysis in Sport, 5(3), 1-11. https://doi.org/10 $.1080 / 24748668.2005 .11868333$

Wright, M. (2014). OR analysis of sporting rules-A survey. European Jour nal of Operational Research, 232(1), 1-8. https://doi.org/10.1016/j. ejor.2013.03.043 